\title{
$\begin{array}{ll}\text { Research Square } & \text { Preprints are preliminary reports that have not undergone peer review. } \\ \text { They should not be considered conclusive, used to inform clinical practice, }\end{array}$
}

\section{Sequential receptor binding primes the SARS-CoV-2 $S$ glycoprotein for membrane fusion}

Donald Benton ( $\square$ donald.benton@crick.ac.uk)

Strutural Biology of Disease Processes Laboratory

Antoni Wrobel ( $\square$ antoni.wrobel@crick.ac.uk)

Strutural Biology of Disease Processes Laboratory

\section{Penqi Xu}

Precision Medicine Center, The Seventh Affiliated Hospital, Sun Yat-sen University, Shenzhen, 518107, Guangdong, China, 1Strutural Biology of Disease Processes Laboratory

\section{Chloë Roustan}

Structural Biology Science Technology Platform;

\section{Stephen Martin}

Strutural Biology of Disease Processes Laboratory

\section{Peter Rosenthal}

Structural Biology of Cells and Viruses Laboratory, Francis Crick Institute, NW1 1AT London, United Kingdom

\section{John Skehel}

Strutural Biology of Disease Processes Laboratory

Steven Gamblin ( $\nabla$ steve.gamblin@crick.ac.uk)

Strutural Biology of Disease Processes Laboratory

\section{Research Article}

Keywords: cryoEM, SARS-CoV-2

Posted Date: July 20th, 2020

DOI: https://doi.org/10.21203/rs.3.rs-45056/v1

License: (c) (i) This work is licensed under a Creative Commons Attribution 4.0 International License. Read Full License

Version of Record: A version of this preprint was published on September 17th, 2020. See the published version at https://doi.org/10.1038/s41586-020-2772-0. 


\section{Abstract}

SARS-CoV-2 infection is initiated by virus binding to ACE2 cell surface receptors, followed by fusion of virus and cell membranes to release the virus genome into the cell. Both receptor binding and membrane fusion activities are mediated by the virus spike glycoprotein, S. As with other class I membrane fusion proteins, $\mathrm{S}$ is post-translationally cleaved, in this case by furin, into $\mathrm{S} 1$ and $\mathrm{S} 2$ components that remain associated following cleavage. Fusion activation following receptor binding is proposed to involve the exposure of a second proteolytic site (S2'), cleavage of which is required for the fusion peptide release. We have investigated the binding of ACE2 to the furin-cleaved form of SARS-CoV-2 S by cryoEM. We classify ten different molecular species including the unbound, closed spike trimer, the fully open ACE2bound trimer, and dissociated monomeric S1 bound to ACE2. The ten structures describe sequential ACE2 binding events which destabilise the spike trimer, progressively opening up, and out, the individual S1 components. The opening process reduces $S 1$ contacts with each other and un-shields the trimeric S2 core, priming fusion activation and dissociation of ACE2-bound S1 monomers. The structures also reveal refolding of one of the S1 subdomains, following ACE2 binding, that disrupts interactions with S2, notably involving Asp614, leading to destabilisation of the structure of S2 proximal to the secondary (S2') cleavage site.

\section{Main Text}

Recognition of the ACE2 receptor by the Spike (S) membrane glycoprotein of SARS- CoV-2 is a major determinant of virus infectivity, pathogenesis, and host range. Previous structural studies on coronavirus S glycoproteins 6,16-21 demonstrated that the $S$ trimer consists of a central helical stalk, made of three interacting S2 components, which is covered at the top by the S1. Each S1 component consists of two large domains, the N-terminal domain (NTD) and receptor-binding domain (RBD), each associated with a smaller intermediate subdomain. In virus membranes, Spike glycoproteins exist in a closed form, in which the RBDs cap the top of the S2 core and are inaccessible to ACE2, and in an open form, in which one S1 component has opened to expose the RBD for ACE2 binding6,16,18,22. Recent structural studies on the isolated RBD of SARS-CoV-2 S in complex with ACE27,23,24 provided a molecular description of the interface. However, even though some comparisons can be inferred from the previous cryoEM studies on $S$ of SARS112,18,19,22, the exact nature and effect of the ACE2 binding to the trimeric $S$ of pandemic SARS-CoV-2 remains unknown.

To examine this interaction between the SARS-CoV-2 spike and its receptor, we mixed ectodomains of furin-cleaved $S$ with ectodomains of ACE2 and incubated them for $\sim 60 \mathrm{~s}$ prior to plunge freezing in liquid ethane for examination by cryoEM. In the images obtained, we could resolve ten distinct species of $S$ and S-ACE2 complexes (Fig. 1 \& S1), ranging from tightly-closed, unbound, trimers to open trimers forming complexes with three ACE2 molecules and dissociated monomeric S1-ACE2 complexes. Of the $S$ trimers analysed, 2/3 have an ACE2 bound (Fig. S1). Of the unbound species, we observe good quality particles in the closed unbound conformation, equally compact to those reported in our previous study 25 and slightly more so than those described in earlier reports 6,16 . There are also significant numbers ( $16 \%$ of all 
trimers) of unbound particles with one erect RBD, as well as some (4\%) in an intermediate conformation, a less compact closed form, with a single disordered RBD, reported in our previous study of furin-cleaved S 25.

Of the $S$ trimers bound to receptor, half accommodate one ACE2 receptor. As reported previously for SARS-CoV-1 S12,22 the ACE2-bound RBD occupies a range of tilts with respect to the long axis of the trimer (Fig. S2A). Of the two RBDs per trimer that do not engage the receptor, either both are closed or one of the RBDs remains closed and one (either clockwise or anticlockwise to the bound S1 [Fig. S1]) is in the open conformation. We were also able to identify, reconstruct, and refine trimers with both two and three ACE2 receptors bound, giving rise to successively more open structures (Fig. 1).

Two points emerge from comparing trimers with one erect RBD that is either bound or unbound by an ACE2 receptor. Firstly, ACE2 binding alters the position of the open RBD by a rigid-body rotation of the domain that moves its centre of mass on average a further $\sim 5.5 \AA$ away from the trimer axis (Fig. S2B) and at the same time the NTDs of all three S1 components move by $1.5-3.0 \AA$ (Fig. S2C). Since bound ACE2 only interacts with the trimer through a single RBD interface this change in the orientation of the bound RBD, and the changes of the NTDs, arise from conformational adjustments that accompany the binding event, although the subtle conformational changes within the RBD that account for this are beyond the resolution of our current maps. Secondly, our data suggest that ACE2 binding favours the open conformation of RBD. The relatively high affinity interaction of RBD with ACE2, generates an $\mathrm{RBD} / \mathrm{ACE} 2$ structure that cannot be accommodated in a closed trimer - the bound state does not have access to the closed conformation. Additionally, the fact that ACE2 binding induces a more open conformation of spike RBD implies that some of the binding energy is used to drive the new conformation of $\mathrm{S} 1$, which is then further excluded from a closed state.

The successive steps, from closed unbound trimer to the fully open, three-ACE2-bound, are associated with a substantive reduction in the contact area each $\mathrm{S} 1$ makes with both its neighbouring $\mathrm{S} 1$ monomers and with the S2 trimeric core (Table S1). For the fully, three- ACE2-bound species, each S1 makes $1400 \AA 2$ less contact with both its S1 trimer neighbours and $1300 \AA 2$ less contact with the S2 core, when compared with the fully-closed trimer conformation; all these rearrangements being driven by the energetics of three ACE2 binding events. The movements of the RBD and NTD domains of S1 associated with the opening of the structure and stabilisation of the new arrangement by ACE2 binding, described above, leaves the trimeric ring of $\mathrm{S} 1$ molecules attached to the $\mathrm{S} 2$ core only by contacts with its two small intermediate subdomains (Fig. 2A). Comparing the ACE2-bound, open form (the open- unbound structure is similar but of poorer local resolution) with the fully-closed trimer, the RBD-associated intermediate subdomain moves about $8 \AA$, while the NTD-associated intermediate subdomain moves by $3 \AA$ (Fig. 2A). The latter also undergoes a partial restructuring with possibly important implications for the mechanism of fusion activation of S. In the closed form, one edge of the NTD-associated intermediate subdomain interacts with a short helix and a loop from S2 of the neighbouring monomer (Fig. 2B). Two particularly noteworthy components of this interaction are a series of side chain $\pi$-stacking interactions in the closed structure25: Tyr636, Phe318 and Arg634 of S1 with Tyr837 of S2; and a salt bridge formed by Asp614 of 
S1 with Lys854 of S2. In contrast, in the ACE2-bound form, Tyr636, Phe318, and Trp633 refold to the side of the domain further away from the symmetry axis (as viewed in Fig. 2C), leaving a channel to accommodate a new segment of a helix that forms downstream of Asp614 from polypeptide chain that was previously disordered. As a consequence, the interactions between S1 and S2 described above for the closed form are lost in the ACE2-bound form and the 827-855 segment of S2 becomes disordered (Fig. 2C). This part of S2 is immediately C-terminal to the putative fusion peptide of S211, the N-terminus of which is defined by Arg815 at the S2' cleavage site9,11. ACE2-stabilised S1 opening therefore leads to destabilisation of $\mathbf{S} 2$ structure just after the putative fusion peptide, potentially activating it for exposure in the next stages of membrane fusion. It is noteworthy that Asp614, which salt bridges to S2 Lys854 in the closed form, is substituted by a glycine residue in the prevalent clade A2a strains of SARS-CoV-21315. Not only would this substitution remove a key salt bridge, but also the unique stereochemistry available to glycine may facilitate the formation of the new segment of a helix, which is also incompatible with the $S 2$ interaction. This amino-acid substitution would lead to reduced stability of the closed form of $\mathrm{S}$, which in turn would increase the likelihood of RBDs adopting the open conformation and hence their ability to bind with ACE2. The suggestion presented here, of enhanced receptor binding and destabilisation of the structure of S2 close to the putative fusion peptide, correlates with the observed selection of the Asp614Gly mutation in SARS-CoV-2.

The opening up, and out, from the trimer axis, of the S1 domains with ACE2 binding gives rise to a sequential un-shielding of the top surface of the helix-loop-helix (approximately residues $980-990$ within the HR1 region20,26) at the top of the S2 domain (Fig. 3). In the closed form, these helices and their connecting turns are tightly shielded by the RBDs; each S2 monomer being predominantly covered by its anticlockwise related S1 trimer neighbour. In the fully open state, the S1 domains move in such a way as to generate a cavity of $50 \AA$ diameter around the trimer axis that is about $65 \AA$ deep. At the bottom of this cavity is the now solvent exposed central portion of HR1. For membrane fusion to occur, by comparison with other class I fusion proteins, as described for the related coronavirus MHV26, the S2 component is likely to undergo a major helical rearrangement, in which the long trimer interface helix 1035:990 grows and extends, by incorporating the refolded turn and helix from the N-terminal portion of HR1, and projects the fusion peptide towards the host cell membrane. In this process, opening up of all three S1 monomers and their subsequent dissociation would enable the concerted helical refolding, since the cooperative displacement of the capping portions of the protein will likely be required for the extension of the helical coil, as recently observed for influenza haemagglutinin 27 .

In addition to the range of species of trimeric $S$ described above, the largest single population of particles we were able to identify and reconstruct represent ACE2 bound to S1 monomer (Fig. 4). The interaction between ACE2 and the RBD, and the interaction of the latter with its associated intermediate subdomain, are very similar between the monomeric and trimer versions and with previously determined crystal and EM structures of RBD and ACE27,23,24. However, there are increasingly large rearrangements between the two intermediate subdomains and then with the NTD. By applying non-uniform refinement, the highest resolution was achieved for the reconstruction of the ACE2/RBD interaction, (Fig. S4), in part because of the tight interaction but also likely because of the dominant influence of this part of the 
structure on the alignment process. Nevertheless, it is clear that there are both increasingly large changes in the interfaces between domains on moving towards the NTD and a range of sub-populations of related but, somewhat variable conformations. The high proportion of ACE2/S1 monomers, and the limited contact areas between the trimeric S1 ring interactions with S2, suggest that the fully open ACE2-bound spike complex is likely metastable.

Taken together, our structural data provide important mechanistic insights into the early stages of SARSCoV-2 infection of cells. SARS-CoV-2 S produces a compact closed form where the helices in the S2 membrane fusion component are fully capped by the RBD of a neighbouring monomer. However, furin cleavage between the S1 and S2 domains enhances the proportion of the trimers able to accommodate one RBD per trimer in an open conformation that is ACE2-binding-competent25. Binding of ACE2 receptor to this open RBD leads to a structural rearrangement within RBD and a more open trimer conformation. The binding of ACE2 is incompatible with the RBD adopting a closed conformation and leads to our observation for SARS-CoV-2 S of several two-open-RBDs conformations, with and without ACE2 bound, and the three-RBD-bound conformation. Successive RBD openings and ACE2 binding lead to a fully open and ACE2-bound form where the trimeric S1 ring remains bound to the core S2 trimer by limited contacts through the subdomains of S1. This arrangement leaves the top of the S2 helices fully exposed. At the same time, the open form of S1 disrupts its interaction with a segment of the $S 2$ chain that precedes the putative fusion peptide region. We suggest that this species is then primed for the helical rearrangements of $\mathrm{S} 2$ required for fusion of the viral and host cell membranes26. Finally, our structure indicates that the Asp614Gly mutation13-15 results in the loss of a salt bridge between S1 and S2 in the closed form. The high prevalence of clade A2a with this mutant correlates with our suggestion that this form of the viral Spike protein could more readily adopt the open conformation required for receptor binding.

\section{Declarations}

\section{Author Contributions}

D.J.B, A.G.W, P.X, C.R, S.R.M performed research, collected and analysed data; A.G.W, D.J.B, P.B.R, J.J.S, S.J.G conceived and designed research and wrote the paper.

\section{Conflict Statement}

We have no conflicts of interest to declare.

\section{Data Availability}

Maps and models have been deposited in the Electron Microscopy Data Bank, http://www.ebi.ac.uk/pdbe/emdb/ (Accession Nos. XXX,XXX,...). Models have been deposited in the 
Protein Data Bank, https://www.ebi.ac.uk/pdbe/ (PDB ID codes XXX, XXX, ...). [Accession numbers will be available before publication].

\section{Acknowledgements}

We would like to acknowledge Andrea Nans of the Structural Biology Science Technology Platform for assistance with data collection, Phil Walker and Andrew Purkiss of the Structural Biology Science Technology Platform and the Scientific Computing Science Technology Platform for computational support. We thank Lesley Calder, Peter Cherepanov, George Kassiotis and Svend Kjaer for discussions. This work was funded by the Francis Crick Institute which receives its core funding from Cancer Research UK (FC001078 and FC001143), the UK Medical Research Council (FC001078 and FC001143), and the Wellcome Trust (FC001078 and FC001143). P.X. was also supported by 100 Top Talents Program of Sun Yat-sen University.

\section{References}

1. Zhou, P. et al. A pneumonia outbreak associated with a new coronavirus of probable bat origin. Nature 579, 270-273 (2020).

2. Wan, Y., Shang, J., Graham, R., Baric, R. S. \& Li, F. Receptor Recognition by the Novel Coronavirus from Wuhan: an Analysis Based on Decade-Long Structural Studies of SARS Coronavirus. Virol. (2020). doi:10.1128/jvi.00127-20

3. Li, , Li, W., Farzan, M. \& Harrison, S. C. Structural biology: Structure of SARS coronavirus spike receptor-binding domain complexed with receptor. Science (80- ). 309, 1864-1868 (2005).

4. Li, W. et al. Angiotensin-converting enzyme 2 is a functional receptor for the SARS coronavirus. Nature 426, 450-454 (2003).

5. Li, F. Structure, Function, and Evolution of Coronavirus Spike Proteins. Rev. Virol. (2016). doi:10.1146/annurev-virology-110615-042301

6. Walls, A. C. et al. Structure, Function, and Antigenicity of the SARS-CoV-2 Spike Glycoprotein. (2020). doi:10.1016/j.cell.2020.02.058

7. Shang, J. et al. Structural basis of receptor recognition by SARS-CoV-2. Nature 1-4 (2020). doi:10.1038/s41586-020-2179-y

8. Belouzard, S., Chu, V. C. \& Whittaker, G. R. Activation of the SARS coronavirus spike protein via sequential proteolytic cleavage at two distinct sites. Natl. Acad. Sci. U. S. A. 106, 5871-5876 (2009).

9. Millet, J. K. \& Whittaker, G. R. Host cell entry of Middle East respiratory syndrome coronavirus after two-step, furin-mediated activation of the spike protein. Natl. Acad. Sci. U. S. A. 111, 15214-15219 (2014). 
10. Hoffmann, M. et al. SARS-CoV-2 Cell Entry Depends on ACE2 and TMPRSS2 and Is Blocked by a Clinically Proven Protease Inhibitor. Cell 181, 271-280.e8 (2020).

11. Lai, A. L., Millet, J. K., Daniel, S., Freed, J. H. \& Whittaker, G. R. The SARS-CoV Fusion Peptide Forms an Extended Bipartite Fusion Platform that Perturbs Membrane Order in a Calcium-Dependent Manner. Mol. Biol. (2017). doi:10.1016/j.jmb.2017.10.017

12. Song, W., Gui, M., Wang, X. \& Xiang, Y. Cryo-EM structure of the SARS coronavirus spike glycoprotein in complex with its host cell receptor ACE2. PLoS Pathog. (2018). doi:10.1371/journal.ppat.1007236

13. Zhang, L. et al. The D614G mutation in the SARS-CoV-2 spike protein reduces S1 shedding and increases infectivity. bioRxiv 06.12.148726 (2020). doi:10.1101/2020.06.12.148726

14. Hu, J. et al. The D614G mutation of SARS-CoV-2 spike protein enhances viral infectivity 1 and decreases neutralization sensitivity to individual convalescent sera 2 Running Title: D614G mutant spike increases SARS-CoV-2 infectivity. bioRxiv (Cold Spring Harbor Laboratory, 2020). doi:10.1101/2020.06.20.161323

15. Korber, B. et al. Spike mutation pipeline reveals the emergence of a more transmissible form of SARS-CoV-2. bioRxiv 04.29.069054 (2020). doi:10.1101/2020.04.29.069054

16. Wrapp, D. et al. Cryo-EM structure of the 2019-nCoV spike in the prefusion. Science (80-. ). (2020). doi:10.1126/science.aax0902

17. Alejandra Tortorici, M. et al. Structural basis for human coronavirus attachment to sialic acid receptors. Struct. Mol. Biol. (2019). doi:10.1038/s41594-019-0233-y

18. Yuan, Y. et al. Cryo-EM structures of MERS-CoV and SARS-CoV spike glycoproteins reveal the dynamic receptor binding domains. Commun. (2017). doi:10.1038/ncomms15092

19. Kirchdoerfer, R. N. et al. Stabilized coronavirus spikes are resistant to conformational changes induced by receptor recognition or proteolysis. Rep. (2018). doi:10.1038/s41598-018-34171-7

20. Pallesen, J. et al. Immunogenicity and structures of a rationally designed prefusion MERS- CoV spike antigen. Natl. Acad. Sci. U. S. A. 114, E7348-E7357 (2017).

21. Walls, A. C. et al. Cryo-electron microscopy structure of a coronavirus spike glycoprotein trimer. Nature(2016). doi:10.1038/nature16988

22. Gui, M. et al. Cryo-electron microscopy structures of the SARS-CoV spike glycoprotein reveal a prerequisite conformational state for receptor binding. Cell Res. (2017). doi:10.1038/cr.2016.152

23. Lan, J. et al. Structure of the SARS-CoV-2 spike receptor-binding domain bound to the ACE2 receptor. Nature 581, 215-220 (2020).

24. Yan, R. et al. Structural basis for the recognition of SARS-CoV-2 by full-length human. Science (80-. ). 367, 1444-1448 (2020).

25. Wrobel, A. et al. SARS-CoV-2 and bat RaTG13 spike glycoprotein structures inform on virus evolution and furin-cleavage effects. Struct. Mol. Biol. In Press (2020). 
26. Walls, A. C. et al. Tectonic conformational changes of a coronavirus spike glycoprotein promote membrane fusion. Natl. Acad. Sci. U. S. A. (2017). doi:10.1073/pnas.1708727114

27. Benton, D. J., Gamblin, S. J., Rosenthal, P. B. \& Skehel, J. J. Structural transitions in influenza haemagglutinin at membrane fusion pH. Nature 583, 150-153 (2020).

\section{Figures}

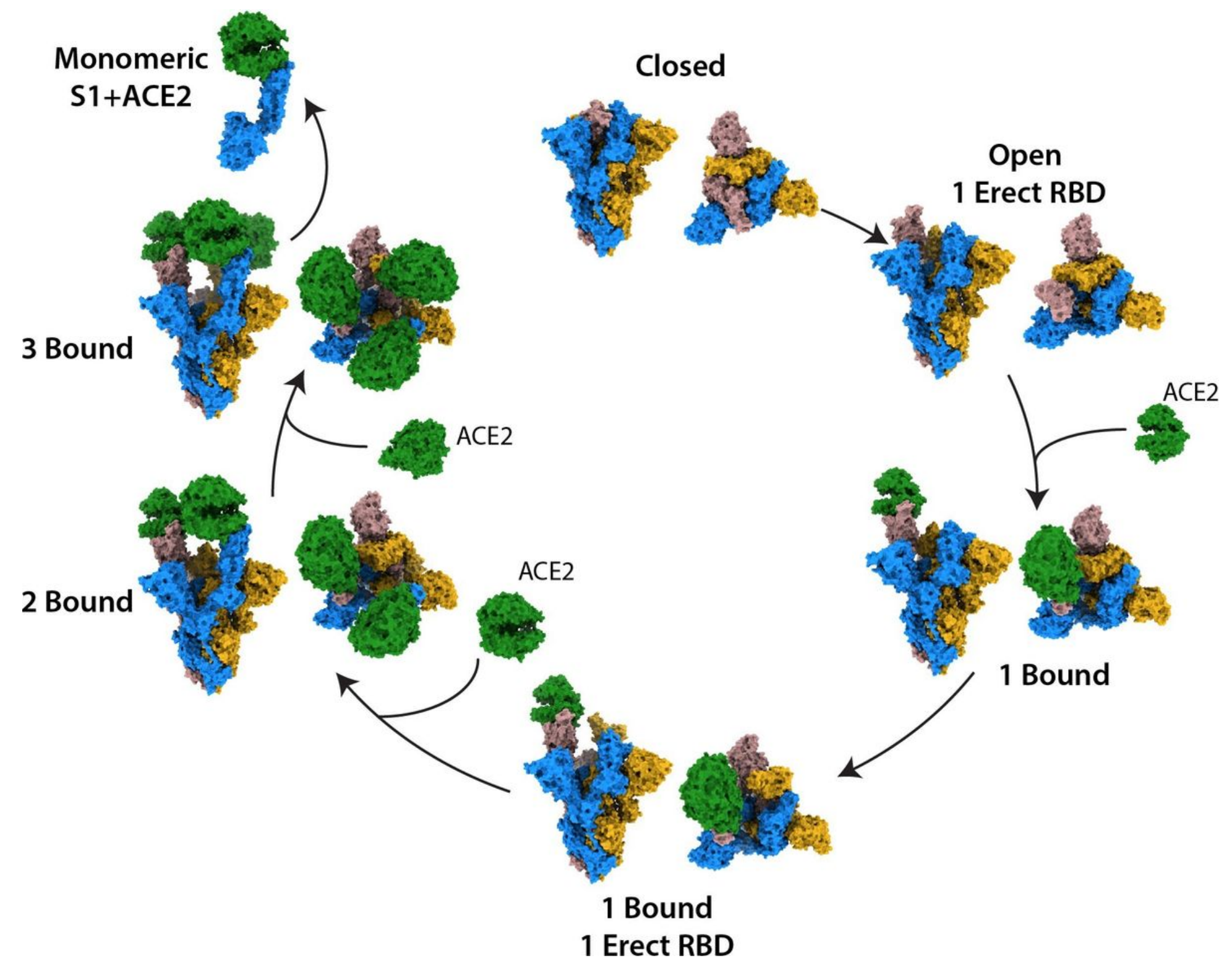

\section{Figure 1}

Sequential steps in ACE2 binding of SARS-CoV-2 S. Surface representation of S, with monomers coloured in blue, brown and gold, and ACE2 coloured in green. Each step shows two views of the S complexes with trimer axis vertical (left) and orthogonal top down view along the axis (right). Clockwise from top we show structures for closed, open but unbound RBD, followed by sequential ACE2 binding events until we 
reach the fully-open, three-ACE2-bound S. From this final trimeric species we show dissociation into monomeric S1-ACE2.

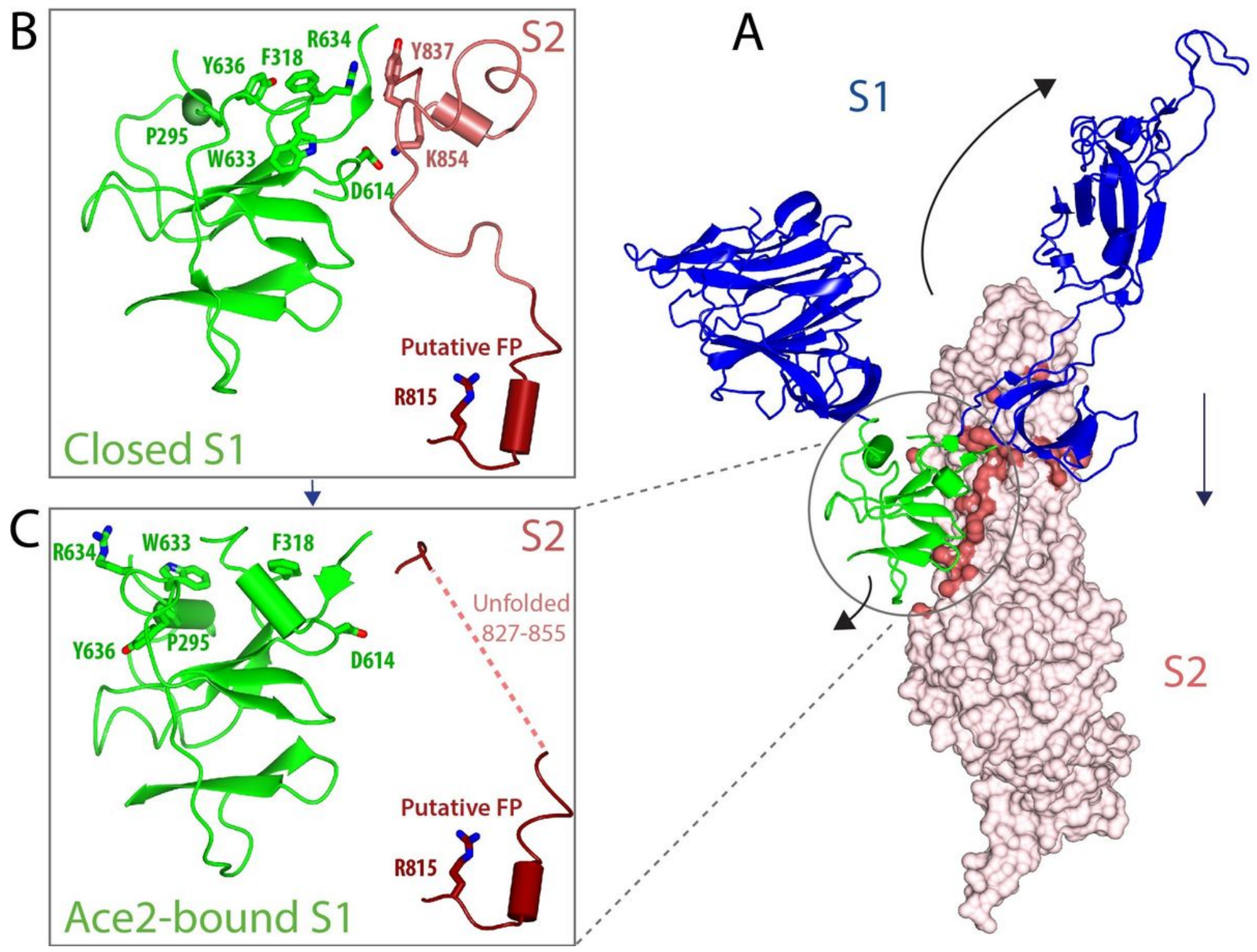

Figure 2

Figure 2. Structural rearrangements between the closed and the ACE2-bound states of S. (A) Surface representation of a monomer of S2 in one-ACE2-bound, two-RBD-closed state coloured in light pink with the S1 domains of the adjacent monomer in ribbon representation; the NTD, RBD-associated subdomain 1 , and RBD in blue and the NTD-associated subdomain 2 in green. The atoms on the surface of S2 contacting the $\mathrm{S} 1$ intermediate domains are coloured in red. The arrows indicate the direction of movements of the intermediate domains, and of the RBD, between the closed and ACE2-bound conformations of S. (B) Ribbons representation of the NTD-associated intermediate domain in green and the moiety of the S2 chain that it interacts with, in red, in the closed conformation of S (PDB 6ZGE, 25). Essential residues participating in the interaction are labelled with the salt bridge between Asp614 (S1, chain A) and Lys854 (S2, chain B) particularly of note. (C) Ribbons representation of the same intermediate domain as in (B) in the conformation observed in the ACE2-bound structure of the spike, where the movement and refolding of the domain leads to a loss of interaction with $\mathrm{S} 2$, which becomes 
disordered. The putative Fusion Peptide (FP) and the S2' site of the second protease cleavage at R815 adjacent to the region that undergoes unfolding are shown in dark red.
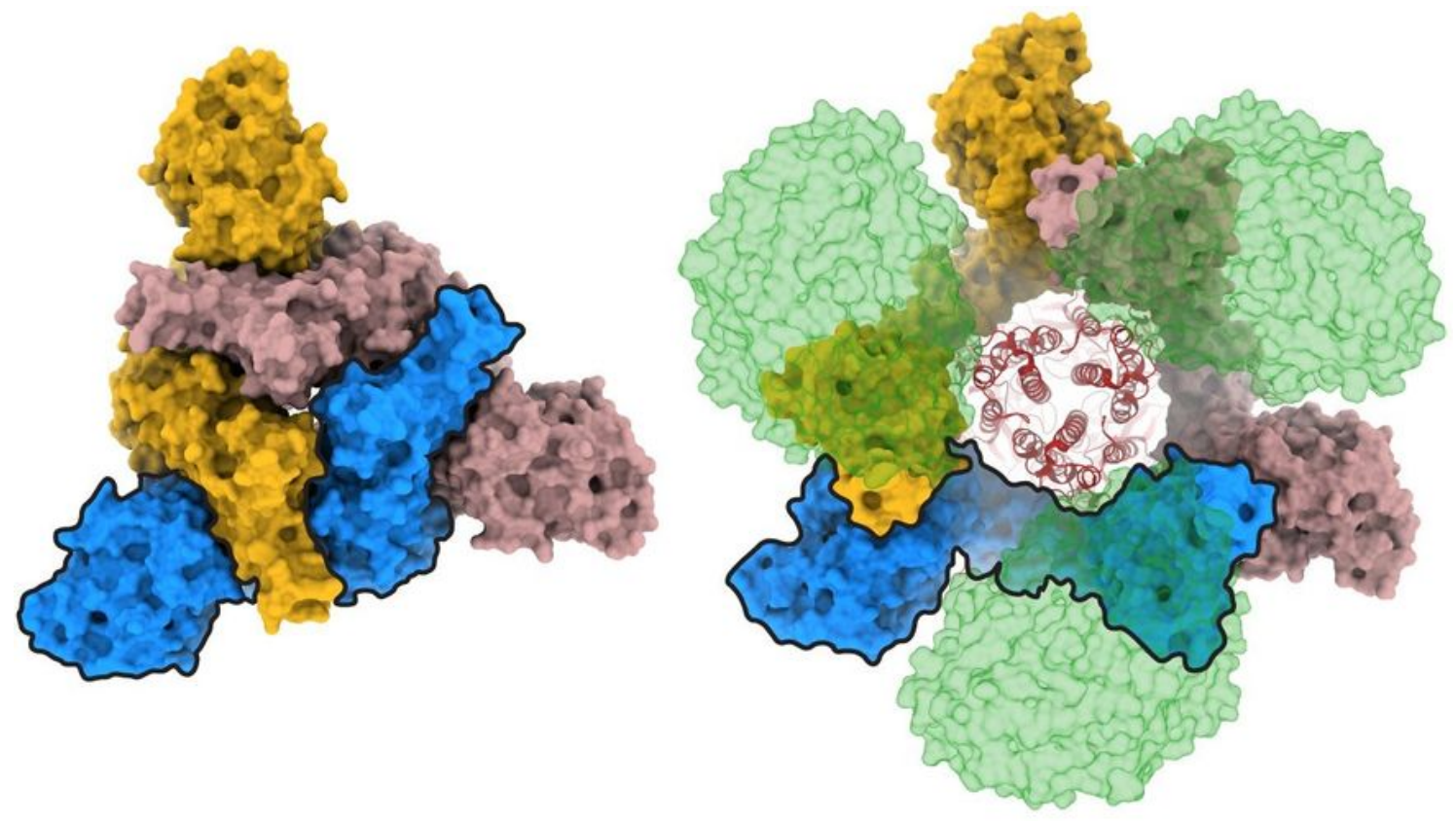

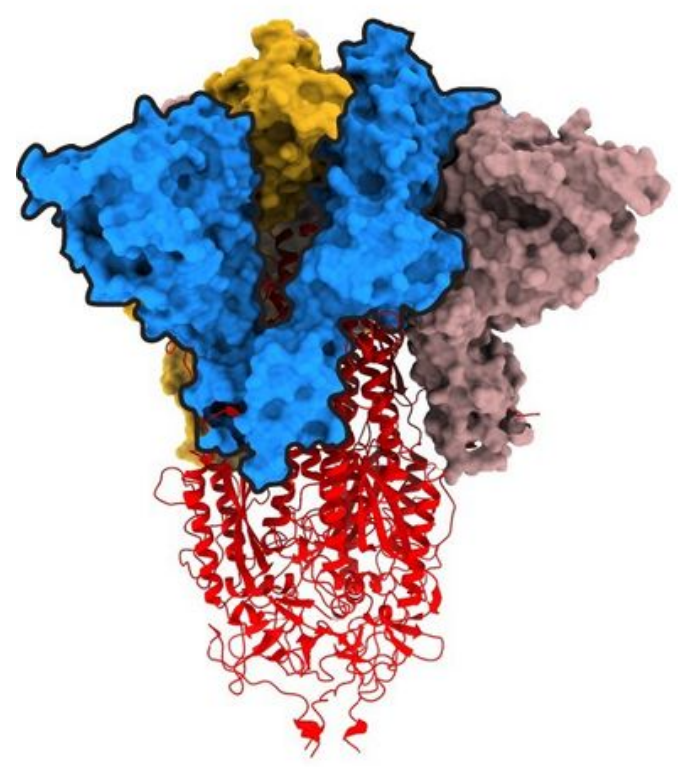

Closed

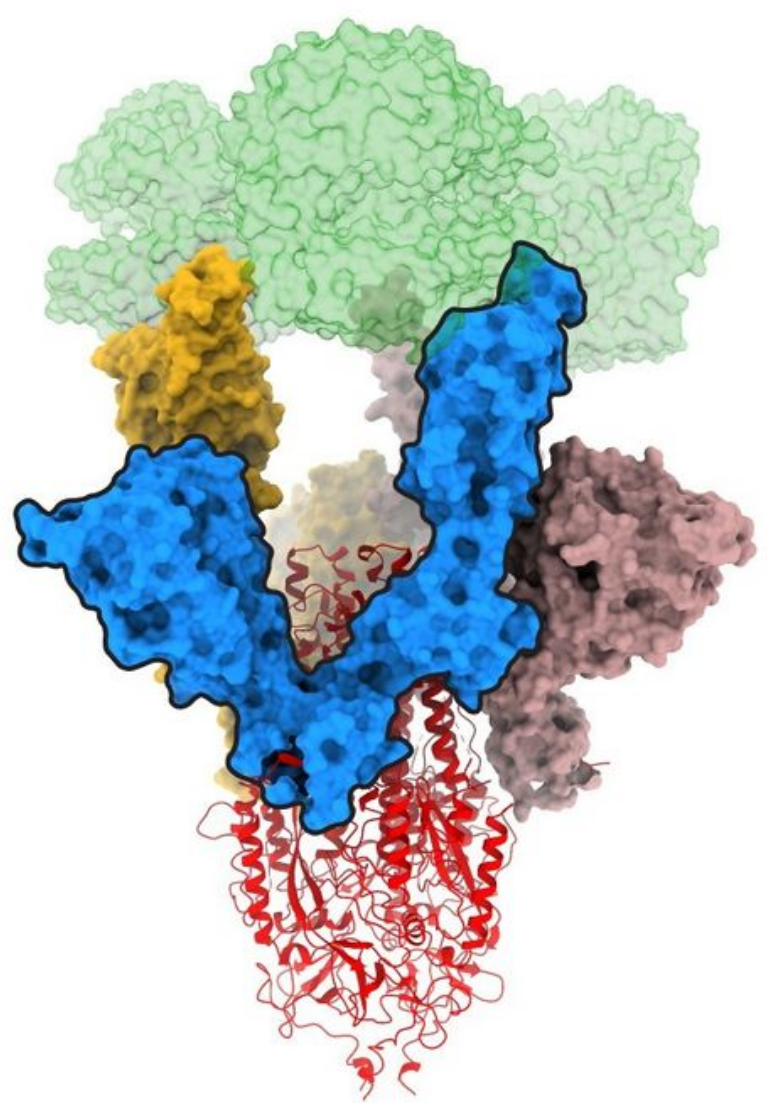

3 ACE2 Bound

\section{Figure 3}

Structural basis of S2 unsheathing by ACE2 binding. S is shown as space filling representation for S1, with each monomer coloured blue, brown, and gold, and as a ribbons representation for $\mathrm{S} 2$ coloured in 
red for all three monomers. The left side shows a top down and side on view of the trimer in the closed conformation, while the right side shows the same views for the fully-open three-ACE2-bound species.
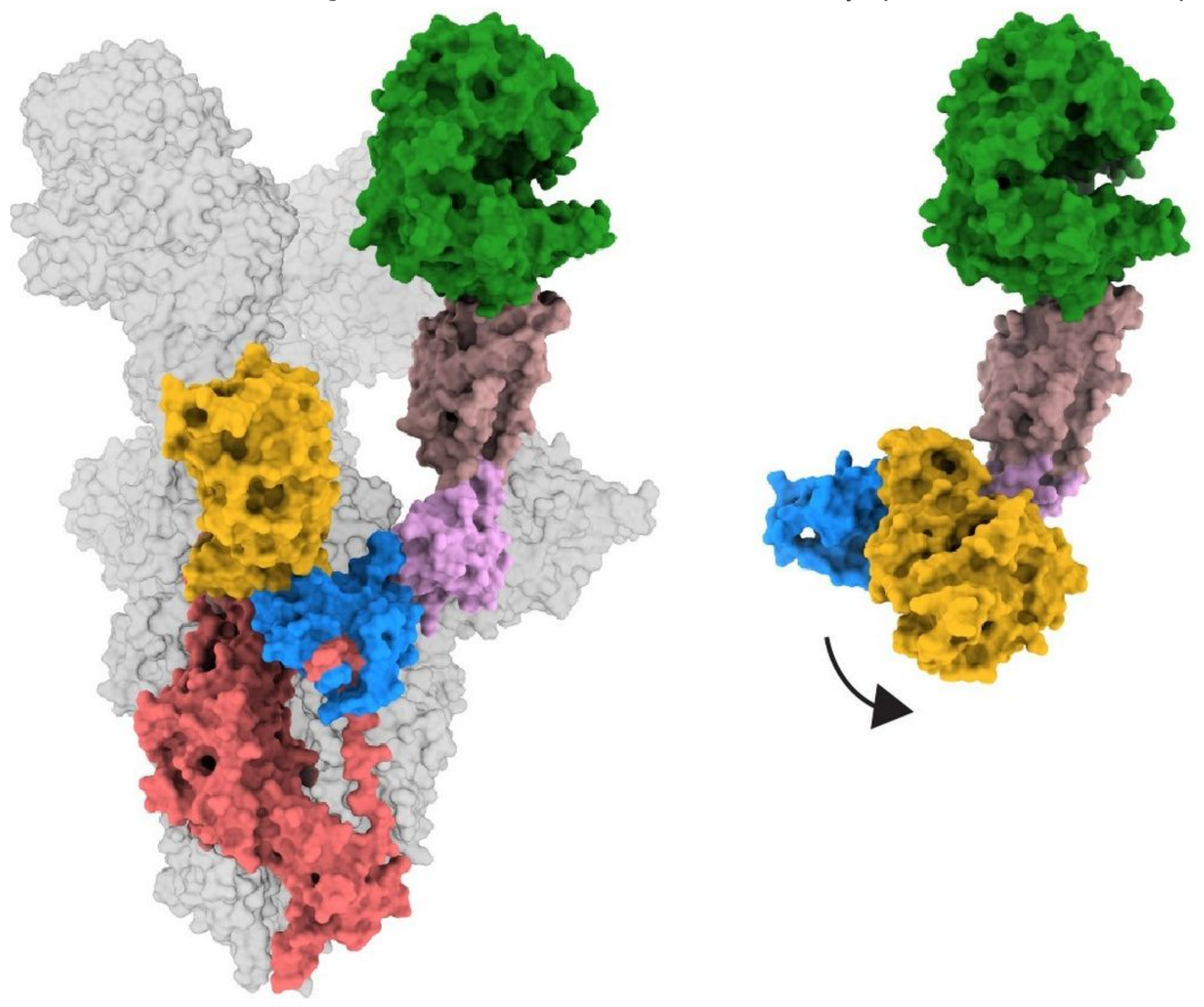

\section{$\mathrm{S} 1+\mathrm{ACE} 2$}

$\mathrm{S} 1+\mathrm{ACE} 2$

Trimer

\section{Dissociated}

\section{Monomer}

Figure 4

ACE2-bound S1 subunit as a part of the $S$ trimer and as an isolated monomer. The right hand panel shows a space filling representation of S with one monomer coloured S1: NTD - yellow, NTD-associated subdomain 2 - blue, RBD-associated subdomain 2 - pink, RBD - brown, S2 - red, ACE2 - green; the rest 
of the trimer is coloured grey. The right-hand panel shows ACE2 bound to monomeric S1 in the same colours. The right hand panel is aligned on the RBD:ACE2 moiety of the trimer complex. The arrow indicates the direction of movement of the NTD and NTD-associated subdomain 2 on the transition from the trimer to the monomer species.

\section{Supplementary Files}

This is a list of supplementary files associated with this preprint. Click to download.

- Supplemental.pdf 